\title{
Stage 3 Neuroblastoma
}

National Cancer Institute

\section{Source}

National Cancer Institute. Stage 3 Neuroblastoma. NCI Thesaurus. Code C6640.

Unresectable unilateral tumor infiltrating across the midline, with or without regional lymph node involvement; or localized unilateral tumor with contralateral regional lymph node involvement; or midline tumor with bilateral extension by infiltration (unresectable) or by lymph node involvement. The midline is defined as the vertebral column. Tumors originating on one side and crossing the midline must infiltrate to or beyond the opposite side of the vertebral column. (cancer.gov) 